\title{
Right diaphragmatic injury and lacerated liver during a penetrating abdominal trauma: case report and brief literature review
}

\author{
Antonino Agrusa*, Giorgio Romano, Daniela Chianetta, Giovanni De Vita, Giuseppe Frazzetta, Giuseppe Di Buono,
} Vincenzo Sorce and Gaspare Gulotta

\begin{abstract}
Introduction: Diaphragmatic injuries are rare consequences of thoracoabdominal trauma and they often occur in association with multiorgan injuries. The diaphragm is a difficult anatomical structure to study with common imaging instruments due to its physiological movement. Thus, diaphragmatic injuries can often be misunderstood and diagnosed only during surgical procedures. Diagnostic delay results in a high rate of mortality.

Methods: We report the management of a clinical case of a 45-old man who came to our observation with a stab wound in the right upper abdomen. The type or length of the knife used as it was extracted from the victim after the fight. CT imaging demonstrated a right hemothorax without pulmonary lesions and parenchymal laceration of the liver with active bleeding. It is observed hemoperitoneum and subdiaphragmatic air in the abdomen, as a bowel perforation. A complete blood count check revealed a decrease in hemoglobin $(7 \mathrm{mg} / \mathrm{dl})$, and therefore it was decided to perform surgery in midline laparotomy.

Conclusion: In countries with a low incidence of inter-personal violence, stab wound diaphragmatic injury is particularly rare, in particular involving the right hemidiaphragm. Diaphragmatic injury may be underestimated due to the presence of concomitant lesions of other organs, to a state of shock and respiratory failure, and to the difficulty of identifying diaphragmatic injuries in the absence of high sensitivity and specific diagnostic instruments. Diagnostic delay causes high mortality with these traumas with insidious symptoms. A diaphragmatic injury should be suspected in the presence of a clinical picture which includes hemothorax, hemoperitoneum, anemia and the presence of subdiaphragmatic air in the abdomen.
\end{abstract}

Keyword: Diaphragmatic injury, Penetrating abdominal trauma, Diaphragmatic repair, Liver laceration, Stab wound

\section{Background}

Diaphragmatic injuries are a diagnostic and therapeutic challenge for the surgeon. They are often un recognized, and diagnostic delay causes high mortality from these injuries [1]. In countries with a low incidence of interpersonal violence, it is quite a rare trauma, with only $4-5 \%$ of patients undergoing laparotomy for trauma presenting a diaphragmatic injury [2]. These are mainly caused by blunt trauma of the chest and abdomen (75\%) and, more rarely, by penetrating ones (25\%) [3]. Clinical presentation

\footnotetext{
*Correspondence: antonino.agrusa@unipa.it

Department of General Surgery, Urgency and Organ Transplantation, University of Palermo, Via L. Giuffrè, Palermo 5 90127, Italy
}

varies from a state of hemodynamic instability secondary to bleeding of the diaphragm and organs involved in the trauma [4] to a condition of intestinal obstruction and respiratory failure that can occur months, or even years, after the trauma, due to diaphragmatic hernia [5]. Diagnosis is made difficult both by the frequent presence of concomitant multi-organ injuries that deviate the surgeon's attention from the diaphragm, and by the lack of adequate diagnostic imaging studies regarding the diaphragmatic muscle. In hemodynamically stable patients with penetrating wound of the abdomen, in which there is a strong suspicion of diaphragmatic injury, with a given negative diagnostic imaging, laparoscopy is considered a valuable 
diagnostic and therapeutic tool in the presence of experienced surgeons. In hemodynamically unstable patients a midline laparotomy is the recommended approach as it allows exploration of the entire abdominal cavity [6].

\section{Methods}

We report the clinical case of a 45 year-old man who came to our observation with a stab wound in the right upper abdomen, without cyanosis or dyspnea. Blood pressure was 130/80 $\mathrm{mmHg}$ and hemoglobin $12.5 \mathrm{mg} / \mathrm{dl}$. On clinical examination, the patient had a lacerated, bleeding stab wound in the right upper quadrant through which part of the omentum, without other macroscopically visible injuries, could be seen. The type or length of the knife used as it was extracted from the victim after the fight. A focused assessment with sonography for trauma (FAST) test was carried out which showed subdiaphragmatic and perihepatic blood. Due to abundant tympanites and lack of cooperation on the part of the patient, nothing more could be seen. It was decided to have to patient undergo a CT scan of the abdomen to determine if there were any lesions to the abdominal organs.

From the scan, the presence of a right hemothorax without pulmonary lesions was seen, with moderate hemoperitoneum from an active bleeding parenchymal liver laceration and subdiaphragmatic air in the abdomen as a bowel perforation (Figure 1). Initially, the suspect of a bowel perforation suggested a laparoscopic approach, but the patient's hemodynamic condition rapidly changed. In the operating room, the patient presented pale with tachycardia; blood pressure decreased to $90 / 60 \mathrm{~mm} \mathrm{Hg}$ and cardiac frequency increased to $115 \mathrm{bpm}$. A complete blood count check revealed a decrease in hemoglobin $(7 \mathrm{mg} / \mathrm{dl})$, and therefore it was decided to perform surgery in midline laparotomy $[6,7]$. After laparotomy, a significant amount of blood was evacuated to identify the site of bleeding. Liver inspection showed an $8 \mathrm{~cm}$ long, $1 \mathrm{~cm}$ deep laceration with active bleeding in segments IV-V (Grade II lesion classification AAST). A careful inspection of the abdominal cavity also showed a $12 \mathrm{~cm}$ length right diaphragmatic lesion with signs of active bleeding that accounted for the presence of free air seen in the CT images. No other intestinal lesions were found. Temporary packing was used to treat the liver bleeding. After evacuating the right hemothorax, we proceeded with repair of the diaphragmatic lesion with nonabsorbable sutures, and by placing a thoracic Bouleau drainage. The suture was completed applying a medicated sponge containing thrombin and human fibrinogen in order to control hemostasis and facilitate the building of the tissues and healing process [8]. After stopping the bleeding from the liver and bile leakage it was decided to adopt a conservative approach applying hemostatic matrix on liver injury (Figure 2). Surgery was concluded with the placement of abdominal drains, in the right subphrenic space. One transfusion was carried out during surgery. In post-operative time, blood pressure was $120 / 80 \mathrm{mmHg}$, hemoglobin $9 \mathrm{mg} / \mathrm{dl}$. Chest tube was removed 4 days post surgery, after an x-ray which confirmed resolution of hemopneumothorax.

\section{Discussion}

The diaphragm is the principle muscle of respiration. With the contraction of striated muscle fibers it carries more than $70 \%$ of the work creating a negative intrathoracic pressure which is necessary for the proper performance of respiratory mechanics, as well as encouraging proper venous return to the heart. The integrity of the diaphragm separates the chest cavity from abdominal positive pressure, which ensures proper maintenance of the different pressure regimes of the two chambers, and prevents the migration of the abdominal organs into the chest. A laceration of the diaphragm produces an alteration of these physiological mechanisms with possible migration of the abdominal viscera into the thorax and the disappearance of the thoraco-abdominal pressure gradient which causes alteration of respiratory mechanics, compression of the vena cava with reduced venous return to the heart, and consequential respiratory failure and cardiovascular collapse [9]. Diaphragmatic rupture is a potentially lethal clinical condition for the patient and a delayed or missed diagnosis causes high mortality with this type of trauma [1].
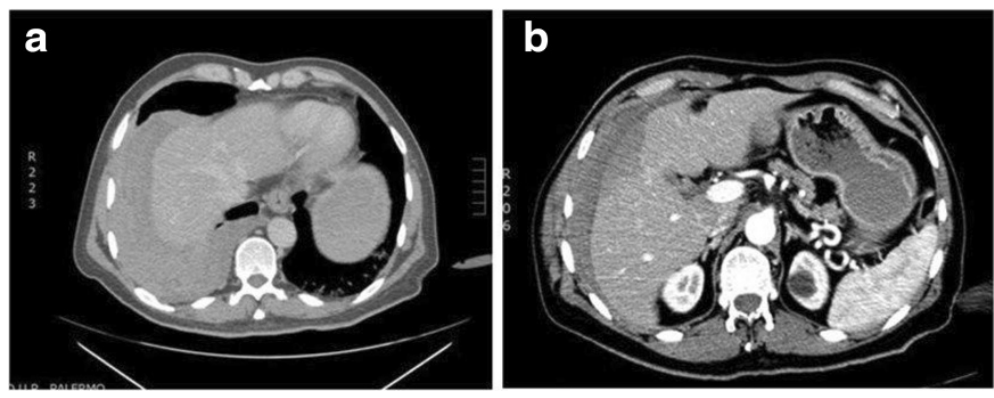

Figure 1 Computed tomography results of the patient. a) presence of a right hemothorax without pulmonary lesions; b) discrete hemoperitoneum by an active bleeding parenchymal liver laceration and "free air" in the abdomen. 


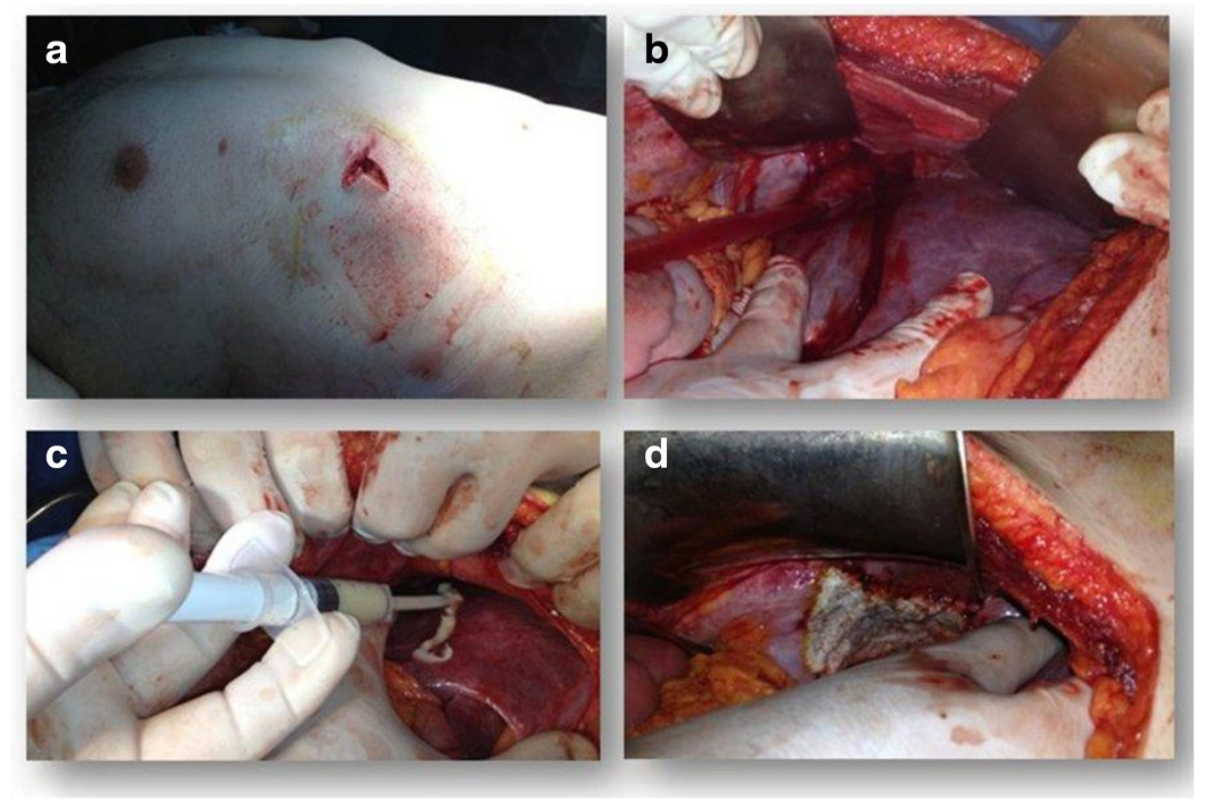

Figure $\mathbf{2}$ Characteristics of the stab wound and intra-operative findings. a) bleeding stab wound in the right upper quadrant; b) liver laceration and right diaphragmatic injury; c) application of hemostatic matrix (Floseal ${ }^{\oplus}$ ) on liver lesion; $\mathbf{d}$ ) repair of diaphragmatic lesion with non-absorbables sutures and positioning of medicated sponge containing thrombin and human fibrinogen (Tachosi ${ }^{\oplus}$ ).

In literature, the first description of diaphragmatic trauma dates back to the sixteenth century when in 1853 Bowditch described a diaphragmatic injury, in a dead victim of a gunshot penetrating trauma, during the autopsy [5]. The first repair with favorable outcomes of a penetrating diaphragmatic injury was described by Riolfi in 1886, while in 1900 Walker published the first repair of traumatic diaphragmatic gunshot lesion with favorable outcomes [10].

It is difficult to accurately estimate the real incidence of diaphragmatic injuries due to delayed or missed diagnosis and pre-hospital deaths [1]. Approximately 5\% of patients with abdominal trauma at the time of thoracotomy or laparotomy have a diaphragmatic injury [2]. They are mainly caused by blunt trauma of the chest and abdomen (75\%) and more rarely by stabbing (25\%) [3]. Diaphragmatic injuries mainly affect the male sex (M/F ratio 3:1) generally occur following closed thoracoabdominal trauma and more rarely penetrating trauma [11]. Mortality rate ranges from $1 \%$ to $28 \%$; this high percentage depends upon frequency of associated injuries but also on the delay between diagnosis and the traumatic event [3]. Diaphragmatic injuries frequently occur during automobile accidents; frontal impact causes an increase of intra-abdominal pressure resulting in a lesion in the radial wall posterolateral to the diaphragm [3]. Side impacts also may be associated with lesions of the liver or spleen in $96 \%$ of cases [11]. Diaphragmatic injuries during penetrating trauma of the abdomen are extremely rare, making up $25 \%$, of which $20 \%$ from gunshot and 5\% from weapon [3]. In the course of penetrating trauma to the abdomen small sized diaphragmatic lesions are often created, which may initially remain undetected and determinate the onset of a diaphragmatic hernia. Right hemidiaphragm trauma is less frequent than left trauma (with a ratio of 1:3) and also is diagnosed with greater delay. This is due to the protective function of the liver which lies on the right abdominal surface preventing herniation of the abdominal viscera into the thorax [9]. Furthermore, many studies performed on cadavers show that during closed trauma the pressure required to determine a lesion of the left hemidiaphragm is less than that required for the right side. [12]. Any discontinuity of the diaphragm leads to alterations of mechanical respiration and circulatory collapse until cardio circulatory system [13]. In severe multiple trauma with patient in a state of shock, respiratory failure and/or coma, diaphragmatic injuries can be misunderstood, as often the attention of the medical team is on damage to other organs which often occur in the course of this type of trauma. In acute phases, diaphragmatic rupture usually occurs with thoracoabdominal pain, hypotension, hemodynamic instability, dyspnea, and cyanosis. Hemodynamic instability and shock are often the result of associated injuries and bleeding of the diaphragmatic muscle injury [14]. When the diaphragmatic lesion is small, it may go unrecognized for several hours, weeks or even months and manifest late and progressively as a diaphragmatic hernia with the appearance of typical symptoms of intestinal obstruction, tachycardia, dyspnea [15]. Small injury of the right hemidiaphragm may even remain 
undetected due to the protective function offered by the liver, which prevents bowel herniation into the thorax cavity. There is rarely herniation of the liver [16]. Preoperative diagnosis of diaphragmatic injury still represents a diagnostic challenge for the radiologist. The high mortality of this trauma is also linked to the difficulty of studying this anatomical site in emergency conditions [1]. In a chest $x$-ray, a diaphragmatic injury should be suspected when the hemidiaphragm is not correctly placed. The specific signs of a diaphragmatic lesion on chest $\mathrm{x}$-rays are represented by the presence of air-fluid levels in the chest and the salience of a hemidiaphragm compared to the contralateral side. Chest $x$-ray has a diagnostic accuracy of less than $40 \%$ and can only detect indirect signs described, the absence of which does not rule out a diaphragmatic lesion [17]. Diagnostic accuracy is four times greater for lesions of the left hemidiaphragm (42\%) compared to the right (17\%) [8]. Chest $\mathrm{x}$-ray has been replaced by computed tomography (CT) which has a diagnostic sensitivity of $50 \%$ for right hemidiaphragm lesions and of $70 \%$ for the left side ones. It allows the physician to see any discontinuity of the diaphragmatic profile and the presence of loops or omentum in the thoracic cavity, as well as the presence of hemoperitoneum and hemothorax [17]. Historically, CT showed poor visualization of the diaphragm due to motion of the muscle itself, but the advent of multiphasic spiral CT has led to a sensitivity of $80 \%$ and a specificity of $90 \%$ [18]. CT is a valuable diagnostic tool, readily available in trauma centers and executable in hemodynamically stable patients with multiple trauma. In hemodynamically unstable patients, ultrasound (US), and in particular FAST in real time can demonstrate the absence or reduced motility of the diaphragm suggestive of lesions of the muscle itself, with an accuracy of $30 \%$. In addition, the US can identify the presence of indirect signs such as hemothorax and hemoperitoneum [19]. Magnetic resonance imaging (MRI) has a sensitivity and specificity of $95 \%$ in identifying a diaphragmatic lesion, it is not always available in emergency rooms, but extremely helpful in the diagnosis of post-traumatic diaphragmatic hernias [20].

Thus, in the absence of a bowel herniation through the lesion, it is very difficult to diagnose a diaphragmatic lesion with the conventional images that are readily available in emergency conditions [21]. This observation is even more valid when penetrating injuries affect the right upper quadrant of the abdomen. In these cases, the liver, due to its particular anatomical position, stands between the lesion and the viscera preventing diaphragmatic herniation of the latter into the chest through the opening in the diaphragm, accounting for the delay in diagnosis of this type of diaphragmatic injury [22]. In this case, there are indirect signs such as effusion into the thorax and abdomen, principally if there is a lacerated liver $(98 \%$ of cases) and the presence of subdiaphragmatic air in the abdomen. In hemodynamically stable patients with penetrating injury of the abdomen in which there is a strong clinical suspicion of diaphragmatic hernia, laparoscopy is indicated as, in addition to having a diagnostic role $[6,23]$ inidentifying the presence of associated lesions, when possible, it also allows repair of the torn diaphragm with a non-absorbable suture sutures [6]. In hemodynamically unstable patients a midline laparotomy is the recommended approach as it allows exploration of the entire abdominal cavity. The diaphragmatic lesion is repaired with non-absorbable suture after placement of chest tube.

In countries with a low incidence of inter-personal violence, stab wound diaphragmatic injury is particularly rare, in particular involving the right hemidiaphragm. Diaphragmatic injury may be underestimated due to the presence of concomitant lesions of other organs, to a state of shock and respiratory failure, and to the difficulty of identifying diaphragmatic injuries in the absence of high sensitivity and specific diagnostic instruments. Diagnostic delay causes high mortality with these traumas with insidious symptoms. A diaphragmatic injury should be suspected in the presence of a clinical picture which includes hemothorax, hemoperitoneum, anemia and the presence of subdiaphragmatic air in the abdomen.

\section{Consent}

Written informed consent was obtained from the patient for publication of this case report and accompanying images. A copy of the written consent is available for review by the Editor-in-Chief of this journal on request.

\section{Competing interests}

The authors declare that they have no competing interests.

\section{Authors' contributions}

AA, RG and CD study design and writing; DVG, FG, DBG and SV data analysis and writing; GG study the design. All authors read and approved the final manuscript.

Authors' information

Agrusa Antonino and other co-authors have no study sponsor.

Received: 27 November 2013 Accepted: 10 April 2014

Published: 28 April 2014

\section{References}

1. Duzgun AP, Ozmen MM, Saylam B, Coskun F: Factors influencing mortality in traumatic ruptures of diaphragm. Ulus Travma Acil Cerrahi Derg 2008, 14(2):132-138.

2. Lewis JD, Starnes SL, Pandalai PK, Huffman LC, Bulcao CF, Pritts TA, Reed MF: Traumatic diaphragmatic injury: experience from a level I trauma center. Surgery 2009, 146(4):578-584.

3. Clarke DL, Greatorex B, Oosthizen GV, Muckart DJ: The spectrum of diaphragmatic injury in a busy metropolitan surgical service. Injury 2009, 40:932-937.

4. Bosanquet $D$, Farboud $A$, Lunckraz $\mathrm{H}$ : A review of diaphragmatic hernia. Resp Med CME 2009, 2:1-6.

5. Bowdich HI: Diaphragmatic hernia. Buffalo Mad J 1853, 9:65-94

6. O'Malley E, Boyle E, O' Callaghan A, Coffey JC, Walsh SR: Role of laparoscopy in penetrating abdominal trauma: a systematic review. World J Surg 2013, 37(1):113-22. 
7. Mattews BD, Bui H, Harold KL, Kervher KW, Adrales G, Park A, Sing RF, Heniford BT: Laparoscopic repair of traumatic diaphragmatic injuries. Surg Endsc 2003, 17:254-258.

8. Toro A, Mannino M, Reale G, Di Carlo I: TachoSil in abdominal surgery: a review. J Blood Med 2011, 2:31-36.

9. RamonVilallonga V, Pastor L, Alvarez R, Charco M, Armengol S, Navarro A: Right-side diaphragmatic rupture alter blunt trauma. An Unusual entity WJES 2011, 6-3.

10. Hedblom CA: Diapragmatic hernia. JAMA 1925, 85:947-953.

11. Morgan BS, Atcyn-Jones TW, Garner GP: Traumatic diaphragmatic injury. J R Army Med Corps 2010, 156(3):139-144.

12. Boulanger BR, Mizman DP, Rosati C, Rodriguez A: A comparison of right and left blunt traumatic diaphragmatic rupture. J Trauma 1993, 35:255-260.

13. Sacco R, Quitadamo S, Rotolo N, Di Nuzzo D, Mucilli F: Traumatic diaphragmatic rupture: personal experience. Acta Bio Medica 2003, 74:71-73.

14. Okada M, Adachi H, Kamesaki M, Mikami M, Ookura Y, Yamakawa J, Hamabe Y: Traumatic diaphragmatic injury: experience from a tertiary emergency medical center. Gen Thorac Cardiovasc Surg 2012, 60:649-654.

15. Goi G, Callegaro D, Villa R, Moroni E, Bondurri A, Danelli P: Large-bowel obstruction as a result of occult diaphragmatic hernia 11 years after injuries. Ann Ital Chir 2012, 83(5):425-428.

16. Kuppusamy A, Ramanathan G, Gurusamy J, Ramamoorthy B, Parasakthi K: Delayed diagnosis of traumatic diaphragmatic rupture with herniation of the liver: a case report. Turk J Trauma Emerg Surg 2012, 18(2):175-177.

17. Matsevych OY: Blunt diaphragmatic rupture: four year's experience. Hernia 2008, 12:73-78.

18. Stein DM, York GB, Boswell S, Shanmuganathan K, Haan M, Scalea TM: Accuracy of computed tomography scan in the detection of penetrating diaphragm injury. J Trauma 2007, 63(3):538-543.

19. Boussuges A, Gole Y, Blanc P: Diaphragmatic motion studied by M-mode ultrasonography: method, reproducibility and normal values. Chest 2009, 135(2):391-400.

20. Sanmuganathan K, Mirvis SE, White CS, Pomerantz SM: MR imagining evaluation of hemidiaphragms in acute blunt trauma: experience with 16 patients. AJR 1996, 167:397-402.

21. Leppaniemi A, Haapiainen R: Occult diaphragmatic injuries causated by stab wouds. J Trauma 2003, 55:646-650

22. Desser TS, Edwards B, Hunt S, Rosenberg J, Purtill MA, Jeffrey JB: The dangling diaphragm sign: sensitivity an comparison with existing $\mathrm{CT}$ signs of blunt traumatic diaphragmatic rupture. Emerg Radiol 2010, 17:37-44.

23. Agrusa A, Romano G, Di Buono G, Dafnomili A, Gulotta G: Laparoscopic approach in abdominal emergiences: a 5-year experience at single centre. G Chir 2012, 33:400-403.

doi:10.1186/1749-7922-9-33

Cite this article as: Agrusa et al:: Right diaphragmatic injury and

lacerated liver during a penetrating abdominal trauma: case report and brief literature review. World Journal of Emergency Surgery 2014 9:33.

\section{Submit your next manuscript to BioMed Central and take full advantage of:}

- Convenient online submission

- Thorough peer review

- No space constraints or color figure charges

- Immediate publication on acceptance

- Inclusion in PubMed, CAS, Scopus and Google Scholar

- Research which is freely available for redistribution 